\title{
Relative corticosteroid insensitivity of alveolar macrophages in severe asthma compared with non-severe asthma
}

\author{
P Bhavsar, M Hew, N Khorasani, A Torrego, P J Barnes, I Adcock, K F Chung
}

Section of Airways Disease, National Heart \& Lung Institute, Imperial College \& Royal Brompton and Harefield NHS Trust Hospital, London, UK

\section{Correspondence to:}

Professor K F Chung, National Heart \& Lung Institute, Imperial College, Dovehouse Street,

London SW3 6LY, UK;

f.chung@imperial.ac.uk

$\mathrm{PB}$ and $\mathrm{MH}$ contributed equally to the work.

Received 4 September 2007 Accepted 19 February 2008 Published Online First 20 May 2008

\begin{abstract}
Background: About 5-10\% of patients with asthma suffer from poorly controlled disease despite corticosteroid (CS) treatment, which may indicate the presence of CS insensitivity. A study was undertaken to determine whether relative CS insensitivity is present in alveolar macrophages from patients with severe asthma and its association with p38 mitogen-activated protein kinase (MAPK) activation and MAPK phosphatase-1 (MKP-1).
\end{abstract}

Methods: Fibreoptic bronchoscopy and bronchoalveolar lavage (BAL) were performed in 20 patients with severe asthma and 19 with non-severe asthma and, for comparison, in 14 normal volunteers. Alveolar macrophages were exposed to lipopolysaccharide (LPS, $10 \mu \mathrm{g}$ / $\mathrm{ml}$ ) and dexamethasone $\left(10^{-8}\right.$ and $\left.10^{-6} \mathrm{M}\right)$.

Supernatants were assayed for cytokines using an ELISAbased method. p38 MAPK activity and MKP-1 messenger RNA expression were assayed in cell extracts.

Results: The inhibition of LPS-induced interleukin (IL)1 $\beta$, IL6, IL8, monocyte chemotactic protein (MCP)-1 and macrophage inflammatory protein (MIP)- $1 \alpha$ release by dexamethasone $\left(10^{-6} \mathrm{M}\right)$ was significantly less in macrophages from patients with severe asthma than in macrophages from patients with non-severe asthma. There was increased p38 MAPK activation in macrophages from patients with severe asthma. MKP-1 expression induced by dexamethasone and LPS, expressed as a ratio of LPS-induced expression, was reduced in severe asthma.

Conclusion: Alveolar macrophages from patients with severe asthma demonstrate CS insensitivity associated with increased p38 MAPK activation that may result from impaired inducibility of MKP-1.

Although most patients with asthma have mild to moderate disease that responds to inhaled corticosteroids (CS), a few have severe disease characterised by asthma symptoms and exacerbations that are largely unresponsive to treatment including systemic CS. ${ }^{1}$ Despite the advent and use of efficacious inhaled CS and long-acting $\beta$-agonists as combination treatment, many patients with asthma do not achieve reasonable control of their asthma. $^{2}$ These patients consume a more significant proportion of medical resources in terms of use of drugs, admissions to hospital or use of emergency services, and time off work or school. ${ }^{34}$ Definitions of severe asthma or treatment-resistant asthma have been proposed by working groups of the European Respiratory and American Thoracic Societies, ${ }^{5}$ and the clinical features of recently described cohorts $^{7-9}$ attest to the persistent loss of control of asthma despite the optimal use of asthma medication.

An important aspect of severe asthma is the relative reduction in effectiveness of CS in controlling asthma. In patients with CS-resistant asthma-defined as a response of $<15 \%$ of baseline forced expiratory volume in $1 \mathrm{~s}\left(\mathrm{FEV}_{1}\right)$ after taking prednisolone (30-40 mg/day) over 14 days while demonstrating marked bronchodilator response to inhaled $\beta_{2}$ agonists $^{10}$ - a reduction in the suppressive effect of dexamethasone on the proliferative response of or release of a neutrophil activating factor from peripheral blood mononuclear cells (PBMCs) has been observed compared with cells from patients with CS-responsive asthma who have $\mathrm{a}>25 \%$ improvement in $\mathrm{FEV}_{1}$ after prednisolone. ${ }^{11} 12$ However, these patients with CSresistant asthma are not similar in clinical presentation or in asthma severity to patients with severe asthma.

The macrophage is an important immune and inflammatory effector cell in asthma and is a potential source of many proinflammatory mediators. The alveolar or airway macrophage is in a greater state of activation in patients with asthma than in individuals without asthma. ${ }^{13-15}$ The alveolar macrophage (AM) may also be an important site of action of CS since inhaled CS therapy causes a reduction in the ability of AMs of patients with mild to moderate asthma to release proinflammatory cytokines. ${ }^{14}$ In a recent investigation we showed that PBMCs obtained from patients with severe asthma demonstrated diminished CS suppression of interleukin (IL)-1 $\beta$, IL8 and macrophage inflammatory protein (MIP)- $1 \alpha$ release relative to $\mathrm{PBMCs}$ from patients with non-severe asthma. ${ }^{16}$

A study was undertaken to determine whether AMs also show similar CS insensitivity in patients with severe asthma. In order to determine the activation status of AMs, we assayed the activity of p38 mitogen-activated protein kinase (MAPK) which is responsive to environmental stresses including heat, osmotic shock, inflammatory cytokines and lipopolysaccharide (LPS). ${ }^{17}$ In addition, p38 MAPK is known to regulate the production of many cytokine proinflammatory cytokines such as IL8 and granulocyte macrophage colony-stimulating factor (GM-CSF) from AMs, ${ }^{18}{ }^{19}$ and overreactivity of p38 MAPK has been associated with CS resistance in PBMCs induced by a combination of IL4 and IL13. ${ }^{20}$ MAPK phosphatase-1 (MKP-1) dephosphorylates and inactivates MAPKs, including p38 $\mathrm{MAPK},{ }^{21}$ and we therefore also measured 
the expression of MKP-1 in the macrophages from patients with severe asthma.

\section{METHODS}

\section{Study participants}

Patients with severe and non-severe asthma were recruited from the cohorts of patients referred to and attending our Asthma Clinic. They demonstrated either an improvement in $\mathrm{FEV}_{1}$ after inhaling $400 \mu \mathrm{g}$ salbutamol from a metered-dose inhaler of $\geqslant 12 \%$ baseline $\mathrm{FEV}_{1}$ or bronchial hyperresponsiveness with a concentration of methacholine provoking a fall in $\mathrm{FEV}_{1}$ of $20 \%$ or more $\left(\mathrm{PC}_{20}\right)$ of $<16 \mathrm{mg} / \mathrm{ml}$. Current and ex-smokers of $>5$ pack-years were excluded.

Patients with severe asthma $(n=20)$ were defined according to the American Thoracic Society major criteria of either continuous or near-continuous oral CS or high-dose inhaled CS or both in order to achieve a level of mild to moderate persistent asthma, and by the presence of two or more minor criteria of asthma control. ${ }^{6}$ These patients underwent a protocol during which the diagnosis of asthma was confirmed, the severity assessed and therapeutic issues were optimised including adherence to treatment, potential aggravating factors assessed and co-morbidity issues evaluated and treated as required. ${ }^{7}$ Patients with non-severe asthma $(n=19)$ were those who did not fall into the severe asthma category and who used 0 $2000 \mu \mathrm{g}$ inhaled beclometasone or equivalent dosage per day with perfect control of their asthma. In order to see how the responses of these patients with asthma compared with those without asthma, 14 healthy volunteers (two women) of mean (SD) age $37.6(2.4)$ years and mean (SD) $\mathrm{FEV}_{1} 98$ (5)\% predicted with no history of asthma, on no medication and who had never smoked were recruited.

The study protocol was approved by the National Heart and Lung Institute and Royal Brompton Hospital Ethics Committee. All volunteers gave written informed consent to participate in the study.

\section{Fibreoptic bronchoscopy}

The entry criteria for performing bronchoscopies in patients with severe asthma were (1) $\mathrm{FEV}_{1} \geqslant 45 \%$ predicted before and $\mathrm{FEV}_{1} \geqslant 55 \%$ predicted following bronchodilators; (2) fewer than six asthma exacerbations in the 6 months before bronchoscopy; (3) no admission to hospital for asthma within 6 months; (4) no endotracheal intubation for asthma within 1 year; and (5) regular daily oral dose of $<20 \mathrm{mg}$ prednisolone.

Fibreoptic bronchoscopy was performed using topical anaesthesia with lignocaine to the upper and lower airways and with intravenous midazolam (3-6 mg) and alfentanyl (125 $\mu \mathrm{g})$. Warmed $0.9 \% \mathrm{NaCl}$ solution was instilled $(50 \mathrm{ml} \times 4)$ in the right middle lobe and bronchoalveolar lavage (BAL) fluid was recovered by gentle suction.

\section{Alveolar macrophage isolation}

Washed BAL cells were suspended in culture media (RPMI with $0.5 \%$ fetal calf serum, antibiotics and L-glutamine) and counted on a haemocytometer. Cytospins were stained with Diff Quick stain (Harleco, Gibbstown, NJ, USA) for differential cell counts. Macrophages were purified by adhesion to the plastic well for $4 \mathrm{~h}$ and then exposed for $18 \mathrm{~h}$ to LPS $(10 \mu \mathrm{g} / \mathrm{ml})$ in the presence or absence of dexamethasone $\left(10^{-8}\right.$ or $\left.10^{-6} \mathrm{M}\right)$. LPS at $10 \mu \mathrm{g} / \mathrm{ml}$ was the submaximal stimulatory concentration in terms of release of IL8.

\section{Cytokine release}

Macrophage supernatants were mixed with microsphere beads (Beadlyte, Upstate Technology, NY, USA) coated with capture antibodies to 10 cytokines: monocyte chemotactic protein (MCP)-1, MIP-1 $\alpha$, Regulated on Activation, Normal T Expressed and Secreted (RANTES), tumour necrosis factor (TNF) $\alpha$, IL1 $\beta$, IL8, interferon (IFN) $\gamma$, IL6, IL10 and GM-CSF. Biotinylated reporter antibodies were added to bind the microsphere bead/cytokine complexes. Finally, a fluorophore streptavidin-phycoerythrin was added to bind the biotinylated reporter, thus emitting a fluorescent signal that was measured in a Luminex 100 laser spectrophotometer (Luminex Corporation, Austin, TX, USA). Microsphere beads for each cytokine emitted a unique ratio of two other fluorophores. Cytokine concentrations were derived from mean fluorescence intensity standard curves.

\section{p38 MAPK phosphorylation}

To determine 38 MAPK activity, additional macrophages from a subset of 10 patients with non-severe asthma and 10 with severe asthma were stimulated with LPS $10 \mu \mathrm{g} / \mathrm{ml}$ for $30 \mathrm{~min}$ in the presence or absence of dexamethasone $\left(10^{-6} \mathrm{M}\right)$ and were scraped. These studies were possible in patients in whom the recovery of macrophages was sufficient. The contents of each well were stored in radioimmunoprecipitation lysis buffer at $-70^{\circ} \mathrm{C}$ for later assay. Maximal p38 MAPK activation occurred at $30 \mathrm{~min}$. Samples were assayed for phosphorylated and total p38 MAK kinase by enzyme immunoassay (Titerzyme, Cambridge Bioscience, UK) using rabbit polyclonal antibodies to phospho-p38 and to total p38.

\section{Real-time RT-PCR for MKP-1}

Macrophages incubated for $24 \mathrm{~h}$ with LPS or with LPS + dexamethasone from the last seven patients with non-severe asthma and the last six patients with severe asthma to be enrolled were used. Total RNA extracts were prepared using RNAqueous-Micro kit (Ambion, Austin, TX, USA).

Table 1 Characteristics of patients with non-severe and severe asthma

\begin{tabular}{|c|c|c|}
\hline & Non-severe asthma & Severe asthma \\
\hline Sex (F:M) & $7: 12$ & $14: 6$ \\
\hline Age (years) & $40.0(2.4)$ & $41.4(2.6)$ \\
\hline Duration of asthma (years) & $27.4(3.2)$ & $27.3(2.6)$ \\
\hline Atopy广 & $18 / 19$ & $18 / 20$ \\
\hline $\mathrm{FEV}_{1}(\%$ predicted) & $84.9(3.0)$ & $59.9(4.7)^{* * *}$ \\
\hline FVC (\% predicted) & $93.9(3.7)$ & $72.9(4.9)^{* * *}$ \\
\hline $\mathrm{FEV}_{1} / \mathrm{FVC}$ ratio & $74.6(2.1)$ & $66.9(2.4)^{*}$ \\
\hline Bronchodilator response & $9.5(1.8)$ & $20.9(3.9)^{* *}$ \\
\hline $\log \mathrm{PC}_{20}(\mathrm{mg} / \mathrm{ml})$ & $0.19(0.14)$ & $-0.48(0.17)^{* *}$ \\
\hline Prednisolone dose (mg/day) & 0 & $11.8(13.7)^{* * *}$ \\
\hline BDP equivalent $(\mu \mathrm{g} /$ day) & $526(167)$ & $2530(292)^{* * *}$ \\
\hline \multicolumn{3}{|l|}{ BAL cells } \\
\hline Total cell count $\left(\times 10^{6}\right)$ & $7.32(0.87)$ & $5.76(0.68)$ \\
\hline Macrophage (\%) & $97.1(0.84)$ & $93.2(1.11)^{* *}$ \\
\hline Lymphocyte (\%) & $1.27(0.34)$ & $2.56(0.63)$ \\
\hline Neutrophils (\%) & $0.98(0.29)$ & $2.58(0.50)^{* *}$ \\
\hline Eosinophils (\%) & $0.63(0.29)$ & $1.73(0.65)$ \\
\hline
\end{tabular}

$\mathrm{BAL}$, bronchoalveolar lavage; BDP, beclometasone dipropionate; $\mathrm{F}$, female; $\mathrm{M}$, male; $\mathrm{FEV}_{1}$, forced expiratory volume in $1 \mathrm{~s}$; $\mathrm{FVC}$, forced vital capacity; $\mathrm{PC}_{20}$, provocative concentration of methacholine causing a $20 \%$ fall in $\mathrm{FEV}_{1}$.

Values are shown as mean (SE).

$\uparrow$ Atopy defined as positive skin prick tests to one or more common aeroallergens. \#Measured as percentage increase in $\mathrm{FEV}_{1}$ after $400 \mu \mathrm{g}$ salbutamol aerosol. ${ }^{*} p<0.05 ;{ }^{* *} p \leqslant 0.01 ;{ }^{* *} p \leqslant 0.001$ compared with non-severe asthma. 

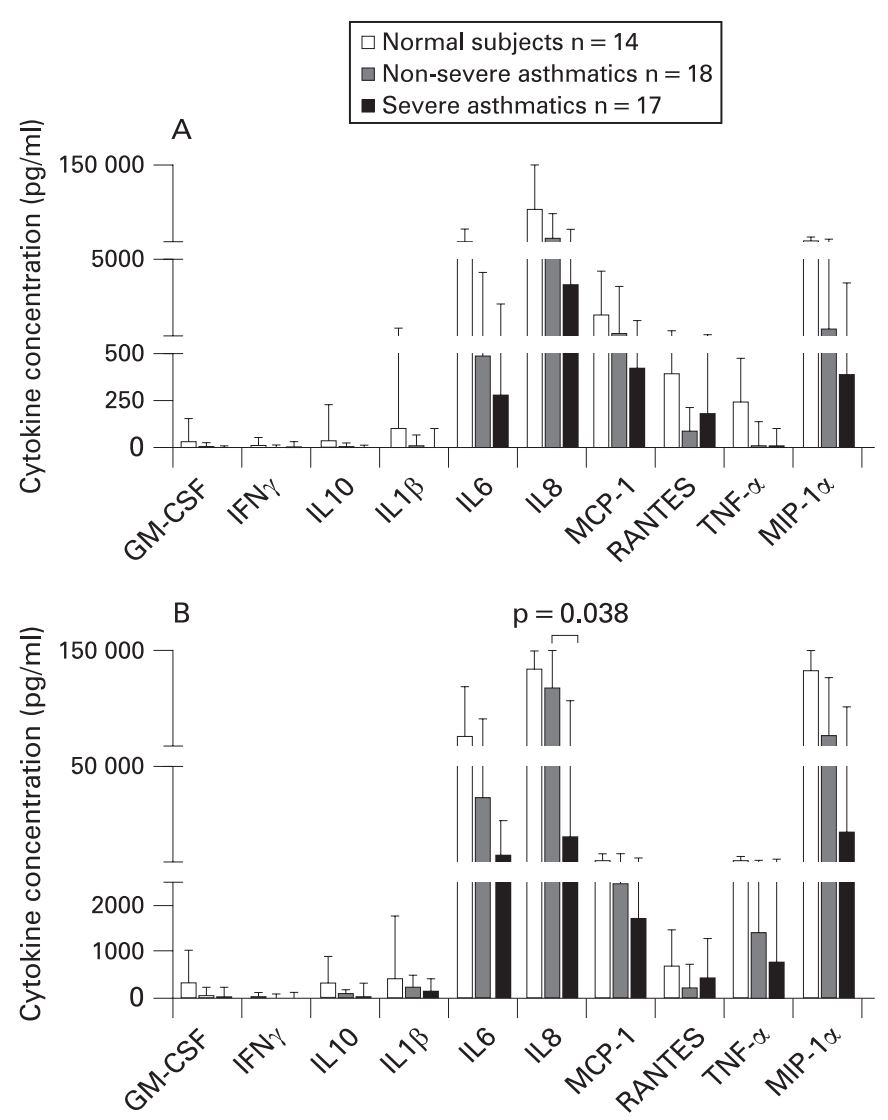

Figure 1 Concentrations of 10 cytokines measured in cell culture supernatants of $(A)$ unstimulated alveolar macrophages and (B) alveolar macrophages stimulated with lipopolysaccharide $10 \mu \mathrm{g} / \mathrm{ml}$ from normal volunteers, patients with non-severe asthma and patients with severe asthma. Data are shown as median (interquartile range). GM-CSF, granulocyte macrophage colony-stimulating factor; IFN $\gamma$, interferon $\gamma$; IL, interleukin; MCP, monocyte chemotactic protein; MIP, macrophage inflammatory protein; RANTES, Regulated on Activation, Normal T Expressed and Secreted; TNF $\alpha$, tumour necrosis factor $\alpha$.

Contaminating genomic DNA was digested with DNase I treatment and DNAse was removed by vortexing with DNAse inactivation resin. The RNA was transferred to an RNAse-free tube and stored at $-20^{\circ} \mathrm{C}$. Complementary DNA (cDNA) for real-time quantitative PCR analysis was synthesised from 500 ng total RNA using random hexamer primers and AMV reverse transcriptase (Promega, Madison, WI, USA). Real-time PCR was performed using the Rotor Gene 3000 (Corbett Research) with PCR amplifications performed in a $20 \mu \mathrm{l}$ reaction volume using the SYBR Green Master Mix Reagent Kit (Promega). Cycling parameters were $95^{\circ} \mathrm{C}$ for $15 \mathrm{~min}$ followed by annealing and extension at 45 cycles of $94^{\circ} \mathrm{C}$ for $15 \mathrm{~s}, 60^{\circ} \mathrm{C}$ for $25 \mathrm{~s}$ and $72^{\circ} \mathrm{C}$ for $25 \mathrm{~s}$. To control for variation within the procedure, a reference mRNA (18 s rRNA) was also measured. Oligonucleotide primer sequences, MKP-1: forward, 5'-GTACATCAAGTCCAT-CTGAC-3'; reverse, 5'-GGTTCTTCTAGGAGTAGACA-3'; 18 s rRNA: forward, 5'-CTTAGAGGGACAAGTGGCG-3'; reverse, 5'-ACGCTGAGCCAGTCAGTGTA-3'.

\section{Data analysis}

The results are expressed as mean (SE). The main statistical analysis pertained to examination of differences between patients with non-severe and severe asthma. Differences in the concentrations of each cytokine released from AMs caused by LPS and by LPS + dexamethasone were calculated and ranked, and a multivariate analysis of variance was performed to determine differences between severe and non-severe asthma. Baseline data between patients with severe and non-severe asthma were compared using the Mann-Whitney $U$ test. Correlations were determined using Spearman rank correlation coefficient. A p value of $<0.05$ was taken as significant.

\section{RESULTS}

\section{Patient characteristics}

Ten patients with severe asthma were on oral prednisolone (5$40 \mathrm{mg} /$ day) and nine of the patients with non-severe asthma were not on inhaled CS. The patients with severe asthma had more airflow obstruction with a lower $\mathrm{FEV}_{1}(\mathrm{p}<0.001)$, greater bronchial hyperresponsiveness $(p<0.05)$ and a greater bronchodilator reversibility $(p<0.01$; table 1$)$. BAL fluid from patients with severe asthma had a higher percentage of neutrophils $(p<0.009)$ and a smaller percentage of macrophages $(p<0.008)$ than BAL fluid from patients with non-severe asthma (table 1).

The bronchoscopic procedure was well tolerated by all subjects. Two of the 20 patients with severe asthma who underwent bronchoscopy developed an asthma exacerbation requiring systemic CS and an extended hospital stay beyond $24 \mathrm{~h}$. Both responded fully to standard asthma treatment.

\section{LPS-stimulated cytokine release}

Baseline release of GM-CSF, IFN- $\gamma$, IL10 and IL1 $\beta$ was close to the limit of detection while, for the other six cytokines, the levels did not differ between patients with severe and those with non-severe asthma (fig 1A). Baseline release of the cytokines was higher than the release observed in the two patient groups. LPS-stimulated cytokine release was also similar between patients with severe and non-severe asthma, except for IL8 release which was greater from AMs of patients with nonsevere asthma (fig 1B).

\section{Corticosteroid suppression of cytokine release}

To quantify the degree of sensitivity of AM to dexamethasone, the release of each cytokine following dexamethasone + LPS stimulation was expressed as a percentage of the cytokine release following LPS stimulation (fig 2). In general, there was less suppression of cytokine release by dexamethasone $10^{-6} \mathrm{M}$ in AMs from patients with severe asthma than from patients with non-severe asthma (fig 2A). Using a multivariate analysis of variance to analyse the suppressed level of each cytokine by dexamethasone $\left(10^{-6} \mathrm{M}\right)$, statistical significance was reached for IL1 $\beta(p=0.05)$, IL6 $(p<0.01)$, IL8 ( $p<0.01)$, MCP-1 $(p<0.05)$ and MIP- $1 \alpha(p=0.01)$. In general, the degree of suppression of cytokine release from macrophages of normal subjects was similar to that of patients with non-severe asthma, apart from IL8. There was no difference in dexamethasone sensitivity between AMs from patients with severe and non-severe asthma at $10^{-8} \mathrm{M}$ (fig $2 \mathrm{~B}$ ), apart from IFN $\gamma$ which was less suppressible in AMs from patients with severe asthma $(p<0.05)$.

\section{LPS-induced p38 phosphorylation}

p38 MAPK phosphorylation, measured as the ratio of phosphorylated p38 to total p38, was increased in AMs from patients with non-severe and severe asthma by LPS, but not in AMs from normal subjects (fig 3A). Baseline p38 MAPK activity of unstimulated AMs was not significantly different between the groups. When expressed as a ratio of p38 phosphorylation after 


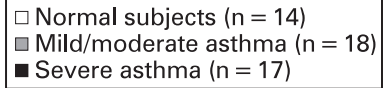

- Severe asthma $(n=17)$
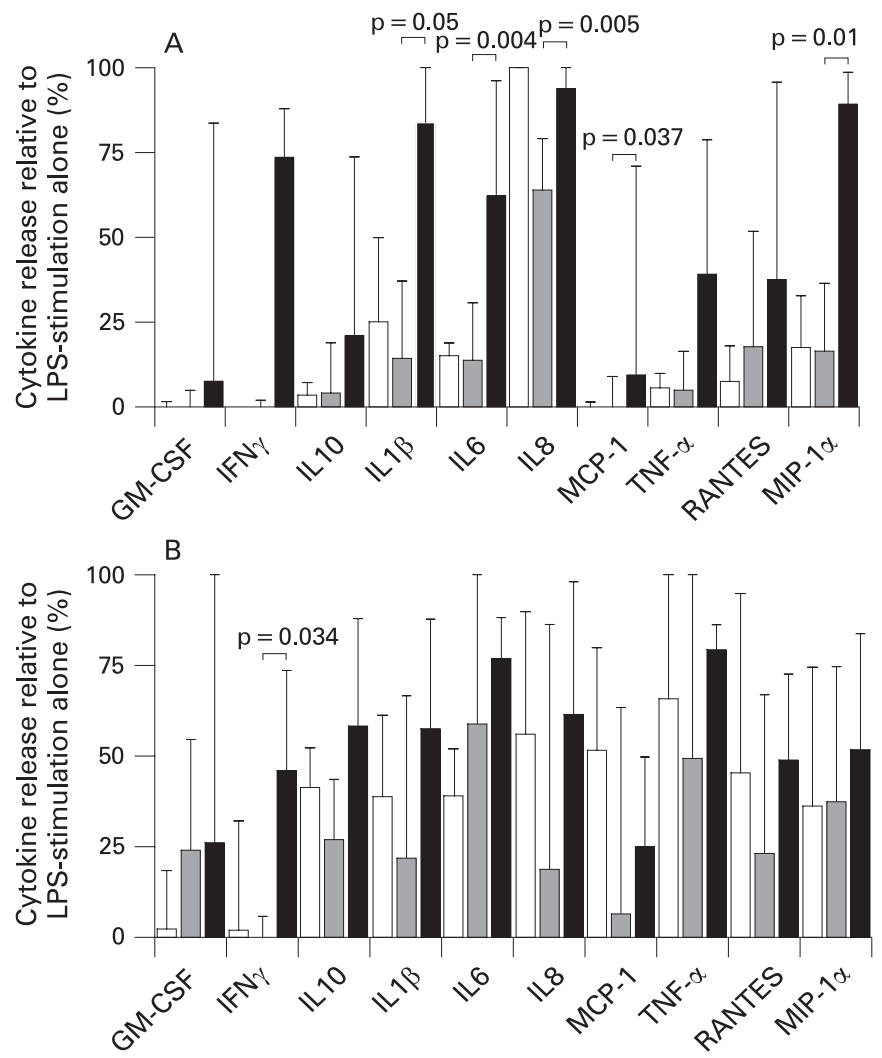

Figure 2 Effect of $(A)$ dexamethasone $10^{-6} \mathrm{M}$ and $(\mathrm{B})$ dexamethasone $10^{-8} \mathrm{M}$ on the suppression of release of 10 cytokines from alveolar macrophages stimulated by lipopolysaccharide (LPS, $10 \mu \mathrm{g} / \mathrm{ml})$. Data are expressed as cytokine release after dexamethasone + LPS as a percentage of release after LPS alone. Data are shown as median (interquartile) range. GM-CSF, granulocyte macrophage colonystimulating factor; IFN $\gamma$, interferon $\gamma$; IL, interleukin; MCP, monocyte chemotactic protein; MIP, macrophage inflammatory protein;

RANTES, Regulated on Activation, Normal T Expressed and Secreted; TNF $\alpha$, tumour necrosis factor $\alpha$.

LPS to unstimulated levels, there was significantly higher activation in AMs from patients with non-severe asthma and severe asthma than those from normal subjects $(p<0.05$ and $\mathrm{p}<0.01$, respectively; fig $3 \mathrm{~B}$ ). However, there was greater activation in patients with severe asthma than in those with non-severe asthma $(\mathrm{p}<0.05)$.

When the data from the two groups of patients with asthma were combined, LPS-induced p38 MAPK phosphorylation correlated directly with the degree of steroid insensitivity of cytokine release for IL1 $\beta(p<0.05)$, IL6 $(p<0.05)$, IL8 $(p<0.05)$ and MIP-1 $\alpha \quad(p<0.01)$ (fig 4). There was no significant correlation for any of the four cytokines in the non-severe asthma group but, in the severe asthma group, there was significant correlation for IL1 $\beta(r=0.76 ; p=0.037)$ and IL6 $(\mathrm{r}=0.7 ; \mathrm{p}=0.043)$.

\section{Induction of MAPK phosphatase-1 (MKP-1) by LPS and dexamethasone}

Induction of MKP-1 gene expression-as measured by mRNA abundance-in AMs stimulated with dexamethasone + LPS was higher than in cells stimulated with LPS alone in normal
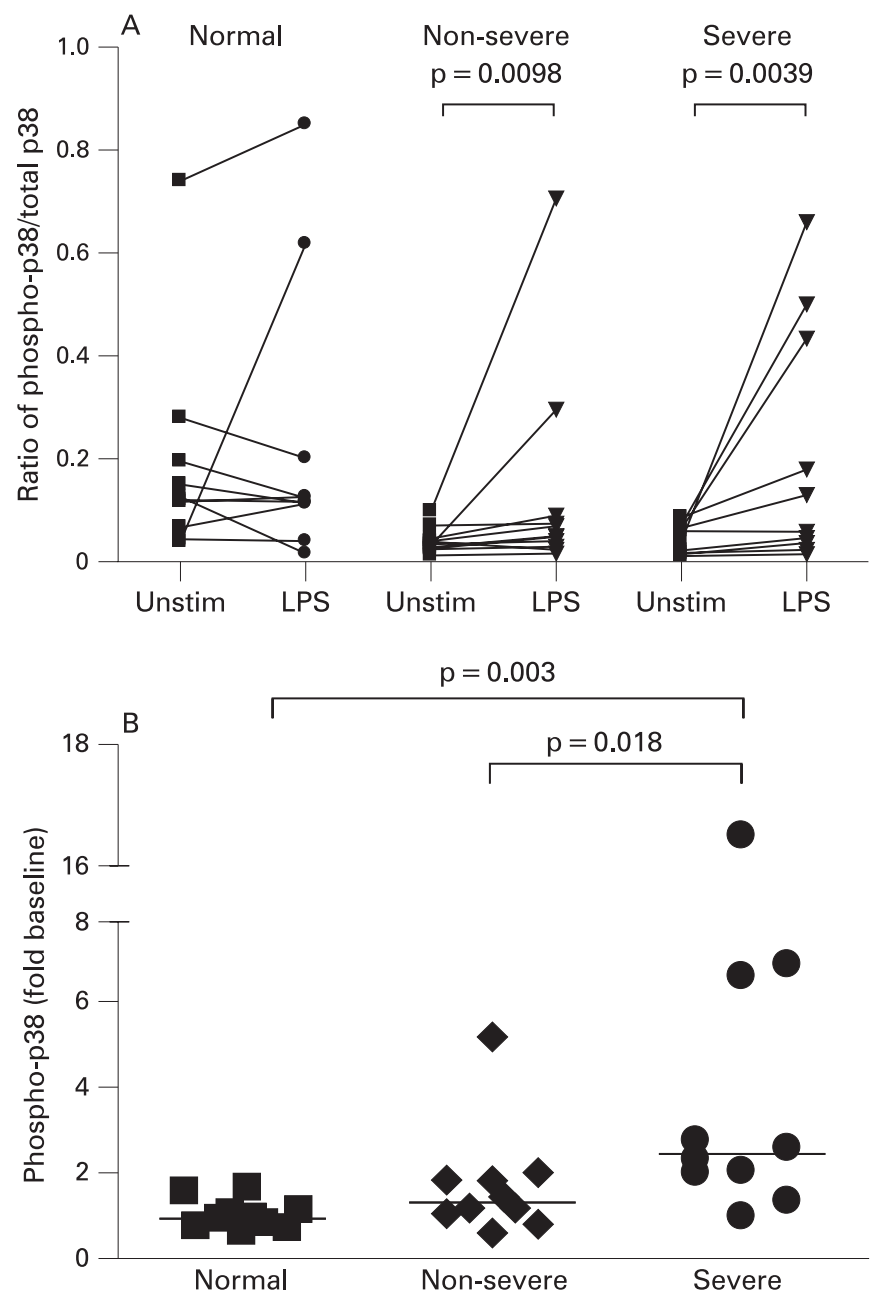

Figure 3 (A) p38 Mitogen-activated protein kinase (MAPK) phosphorylation measured as the ratio of phosphorylated p38 to total p38 at baseline (unstim) and following stimulation by lipopolysaccharide (LPS; $10 \mu \mathrm{g} / \mathrm{ml}$ ) in alveolar macrophages from normal subjects and those with severe and non-severe asthma. (B) Similar data expressed as the fold increase in p38 MAPK phosphorylation after LPS compared with baseline. Horizontal bars are median values.

subjects and patients with non-severe asthma $(\mathrm{p}<0.01$ and $\mathrm{p}<0.05$, respectively). However, this increase in MKP-1 mRNA abundance was not observed in AMs from patients with severe asthma. Baseline transcript levels of MKP-1 were not significantly different between the three groups. MKP-1 mRNA expression following treatment with dexamethasone + LPS compared with that induced by LPS alone was significantly higher in patients with non-severe asthma than in those with severe asthma $(p<0.05$; fig 5$)$.

\section{DISCUSSION}

AMs from patients with severe asthma demonstrate impaired CS suppression of release of several inflammatory cytokines (IL1 $\beta$, IL6, IL8, MCP-1 and MIP-1 $\alpha$ ) stimulated by LPS compared with AMs from patients with non-severe asthma. CS sensitivity of AMs from patients with non-severe asthma paralleled that of AMs from subjects without asthma. The differences between patients with severe and non-severe asthma were seen with dexamethasone $10^{-6} \mathrm{M}$ but not with dexamethasone $10^{-8} \mathrm{M}$, indicating a reduction in maximal CS response of the former group. Our results are therefore similar 
Figure 4 Correlation between relative steroid suppression of $(A)$ interleukin (IL) $1 \beta$, (B) IL6, (C) IL8 and (D) macrophage inflammatory protein (MIP)$1 \alpha$ release and the increase in p38 phosphorylation induced by lipopolysaccharide (LPS) in alveolar macrophages of patients with asthma. Closed circles represent patients with severe asthma and open circles represent patients with non-severe asthma. Dex, dexamethasone.
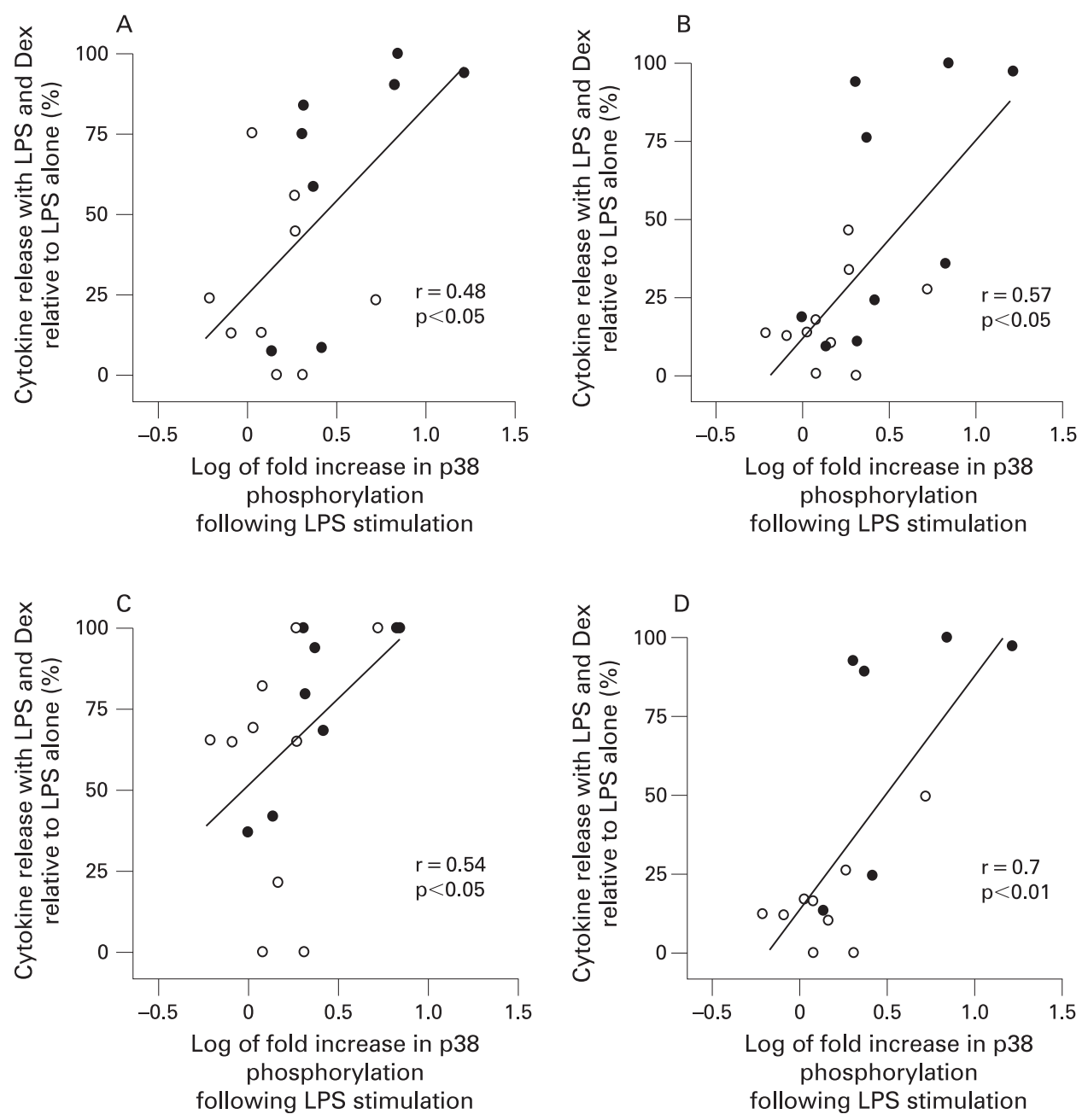

to those we previously reported in PBMCs obtained from patients with severe asthma. ${ }^{16}$ In the current study we examined the possibility that the decrease in CS sensitivity of the AMs in severe asthma was related to increased activation of p38 MAPK phosphorylation. We found that p38 MAPK activation was increased in the severe asthma group than in the non-severe asthma and normal groups. Furthermore, the degree of CS insensitivity was correlated with the degree of p38 MAPK activation, providing support for p38 MAPK activation as a potential determinant of CS responsiveness.

Although there was increased activation of p38 MAPK in AMs from patients with severe asthma, we did not find any increase in cytokine release induced by LPS in these patients. In addition, there was only a relatively small difference in inflammatory markers between the two groups, with a significant increase in neutrophils in BAL fluid from patients with severe asthma, despite the fact that these patients were being treated with higher doses of inhaled CS and $50 \%$ of them were also taking a daily dose of prednisolone. This may be a reflection of the relative reduction in CS sensitivity observed in patients with severe asthma. The effect of dexamethasone in inhibiting cytokine release was impaired, an observation that was associated with relative underexpression of MKP-1 induced by dexamethasone. This indicates that there may be a defect in MKP-1 expression, which is known to be induced by CS, ${ }^{22}$ that may allow for excessive p38 MAPK activity. Macrophage cell lines transfected with MKP-1 show an accelerated inactivation of p38 MAPK, ${ }^{23}$ and murine AMs treated with the MKP-1 inhibitor triptolide show prolonged p38 activity. ${ }^{24}$ Similarly, dexamethasone-mediated suppression of the proinflammatory cytokines TNF $\alpha$ and IL1 $\beta$ was impaired in AMs from MKP-1 knock-out mice, indicating that the expression of MKP-1 was required for inhibition of these cytokines by dexamethasone. ${ }^{25}$ The inability of CS to upregulate MKP-1 activity in severe asthma could be a mechanism by which CS insensitivity occurs, since some of the biological effects of CS may occur through inactivation of MAPK kinases. ${ }^{26}$ However, AMs from the group with non-severe asthma also demonstrated a reduction in MKP1 expression in the presence of dexamethasone, although to a lesser extent than AMs from subjects with severe asthma, indicating that the asthmatic state itself is associated with an inability of MKP-1 upregulation. However, given the small number of asthmatic samples examined, the data on MKP-1 should be considered preliminary and needs to be confirmed in larger studies.

The fact that patients with severe asthma still have uncontrolled asthma despite taking high doses of inhaled CS-sometimes together with oral CS-has led to the hypothesis that patients with severe asthma are relatively resistant to the therapeutic effect of CS. Such patients are not absolutely resistant to the effects of CS since stopping CS therapy usually leads to a worsening of asthma in these patients. Rather, such patients, often labelled as "corticosteroid-dependent asthma", have a partial impaired response to CS. This current work on AM activation studied ex vivo provides some support for this concept. Recent studies of cohorts of patients with severe 

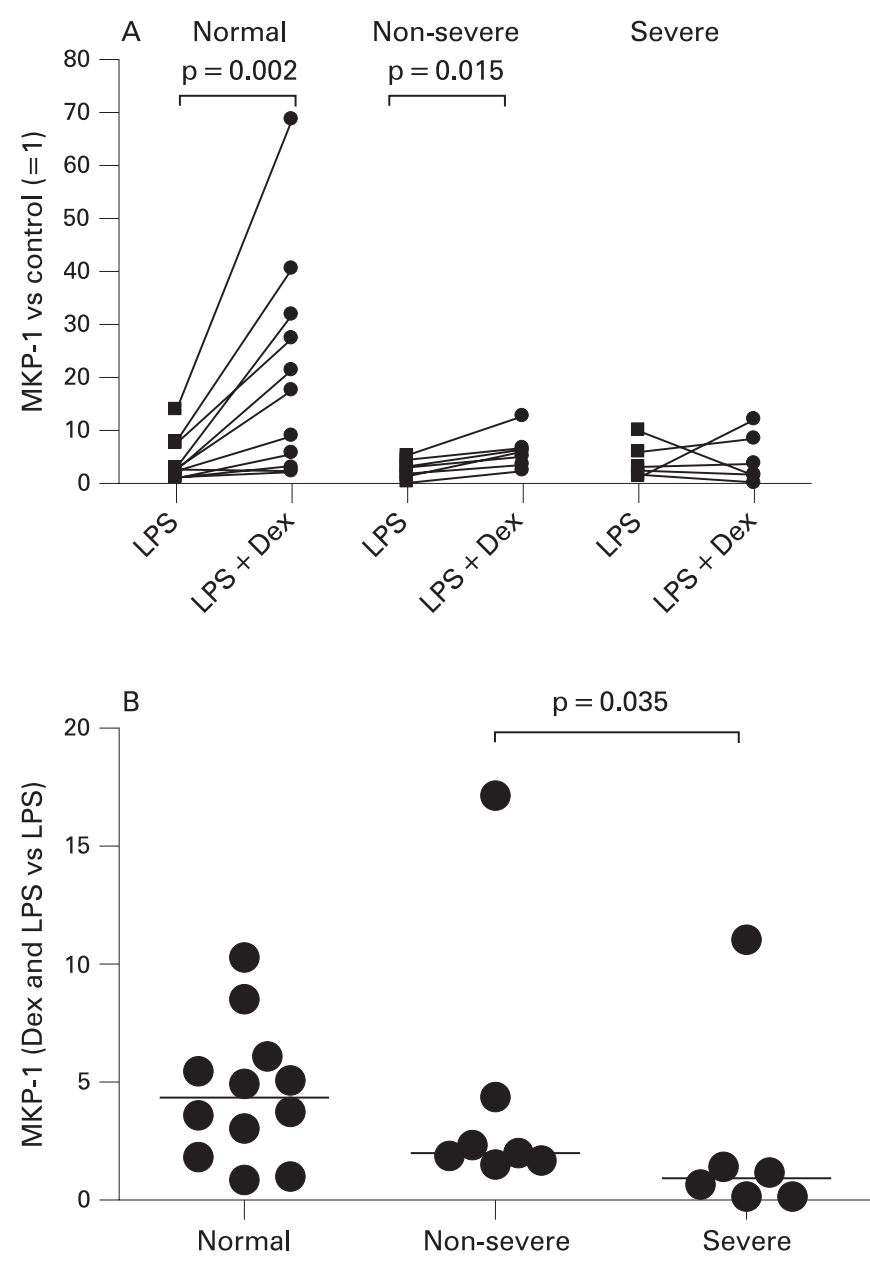

Figure 5 Induction of MAPK phosphatase-1 (MKP-1) mRNA expression measured by quantitative RT-PCR. (A) Effect of lipopolysaccharide (LPS) alone and LPS + dexamethasone (Dex $\left.10^{-6} \mathrm{M}\right)$. (B) Ratio of expression after LPS + dexamethasone to that after LPS alone in alveolar macrophages from normal volunteers and patients with non-severe and severe asthma. Horizontal bars are median values.

asthma have revealed that these patients show daily symptoms and increased use of reliever medication, increased urgent healthcare utilisation with recurrent exacerbations of asthma, and a substantial proportion of patients on a regular oral dose of prednisolone. $^{89}$ Patients with severe asthma recruited to the current study had a greater degree of airflow obstruction, a greater bronchodilator response to salbutamol and a greater degree of methacholine airway responsiveness than patients with non-severe asthma, all indicators of asthma severity. However, in terms of inflammation, there was only a small increase of 2.5-fold in BAL fluid neutrophils. Airway neutrophilia has been recognised as a feature of severe asthma, ${ }^{27}{ }^{28}$ although this could be a reflection of oral CS therapy. ${ }^{29}$ One might, indeed, impute the reduced CS sensitivity in patients with severe asthma to the continuous use of CS, particularly oral CS, that could lead to downregulation of the CS receptor expression and translocation. However, we found no differences in dexamethasone suppression of AM function from patients with severe asthma on inhaled CS alone and those on inhaled and oral CS therapy. Similarly, there were no differences in AM sensitivity from patients with non-severe asthma on inhaled CS and those not taking inhaled CS.
We have previously shown that IL2 and IL4 exposure of blood monocytes caused a reduction in CS ligand binding affinity due to phosphorylation of the glucocorticoid receptor that could be reversed by a p38 MAPK inhibitor ${ }^{20}$ or by an indirect effect on the ligand binding domain of glucocorticoid receptor. ${ }^{30}$ p38 MAPK activation may also lead to phosphorylation and phosphoacetylation of histones at the promoter regions of NF$\kappa \mathrm{B}$ dependent genes such as those activated by LPS, resulting in enhanced recruitment of the transcription factor NF- $\kappa B^{19}{ }^{31}$ This is consistent with our previous observation that histone deacetylase (HDAC) activity is reduced in PBMCs and AMs from patients with asthma. ${ }^{16}{ }^{32}$ p38 MAPK activation may be involved in the stabilisation and increased translation of proinflammatory cytokine mRNA, dependent on the conserved AU-rich elements in the $3^{\prime}$-UTR region, ${ }^{33}$ particularly IL1 $\beta$, IL6, IL8 and MIP- $1 \alpha \cdot{ }^{34-36}$ These potential downstream effects of p38 MAPK may lead to a reduction in the effectiveness of dexamethasone.

In summary, AMs from patients with severe asthma show relative CS insensitivity compared with those from patients with non-severe asthma. Increased p38 MAPK activation may underlie the insensitivity, but further work will be needed to implicate p38 MAPK activation directly in the CS insensitivity of severe asthma.

Acknowledgements: The authors thank Sally Meah for recruitment of patients and for help with the bronchoscopic procedures.

Funding: This work was supported by NIH R0-1 grant HL-69155 and was conducted within the Severe Asthma Research Program (SARP) whose principal investigators were E Israel, Brigham and Women's Hospital; SC Erzurum, Cleveland Clinic; WG Teague, Emory University; KF Chung, Imperial College London; SE Wenzel, National Jewish Medical and Research Center; WJ Calhoun, University of Texas; B Gaston, University of Virginia; WW Busse, University of Wisconsin; ER Bleecker, Wake Forest University; M Castro, Washington University in St Louis.

Competing interests: None.

Ethics approval: The study protocol was approved by the National Heart and Lung Institute and Royal Brompton Hospital Ethics Committee. All volunteers gave written informed consent to participate in the study.

\section{REFERENCES}

1. Stirling RG, Chung KF. Severe asthma: definition and mechanisms. Allergy 2001;55:1-16.

2. Bateman ED, Boushey HA, Bousquet J, et al. Can guideline-defined asthma control be achieved? The Gaining Optimal Asthma ControL study. Am J Respir Crit Care Med 2004;170:836-44.

3. Dolan CM, Fraher KE, Bleecker ER, et al. Design and baseline characteristics of the epidemiology and natural history of asthma: Outcomes and Treatment Regimens (TENOR) study: a large cohort of patients with severe or difficult-to-treat asthma. Ann Allergy Asthma Immunol 2004;92:32-9.

4. Godard $\mathbf{P}$, Chanez $P$, Siraudin L, et al. Costs of asthma are correlated with severity: a 1-yr prospective study. Eur Respir J 2002;19:61-7.

5. Chung KF, Godard P, Adelroth E, et al. Difficult/therapy-resistant asthma: the need for an integrated approach to define clinical phenotypes, evaluate risk factors, understand pathophysiology and find novel therapies. ERS Task Force on Difficult/ Therapy-Resistant Asthma. European Respiratory Society. Eur Respir J 1999;13:1198-208

6. American Thoracic Society. Proceedings of the ATS workshop on refractory asthma: current understanding, recommendations, and unanswered questions. Am J Respir Crit Care Med 2000;162:2341-51.

7. Robinson DS, Campbell DA, Durham SR, et al. Systematic assessment of difficultto-treat asthma. Eur Respir J 2003;22:478-83.

8. European Network for Understanding Mechanisms of Severe Asthma. The ENFUMOSA cross-sectional European multicentre study of the clinical phenotype of chronic severe asthma. Eur Respir J 2003;22:470-7.

9. Moore WC, Bleecker ER, Curran-Everett D, et al. Characterization of the severe asthma phenotype by the National Heart, Lung, and Blood Institute's Severe Asthma Research Program. J Allergy Clin Immunol 2007;119:405-13.

10. Woolcock AJ. Corticosteroid-resistant asthma. Definitions. Am J Respir Crit Care Med 1996;154(2 Pt 2):S45-8.

11. Corrigan J, Brown PH, Barnes NC, et al. Glucocorticoid resistance in chronic asthma Am Rev Respir Dis 1991;144:1016-25. 
12. Wilkinson JRW, Crea AEG, Clark TJH, et al. Identification and characterization of a monocyte-derived neutrophil-activating factor in corticosteroid-resistant bronchial asthma. J Clin Invest 1989;84:1930-41.

13. Viksman MY, Liu MC, Bickel CA, et al. Phenotypic analysis of alveolar macrophages and monocytes in allergic airway inflammation. I. Evidence for activation of alveolar macrophages, but not peripheral blood monocytes, in subjects with allergic rhinitis and asthma. Am J Respir Crit Care Med 1997;155:858-63.

14. John M, Lim S, Seybold J, et al. Inhaled corticosteroids increase interleukin-10 but reduce macrophage inflammatory protein-1a, granulocyte-macrophage colonystimulating factor and interferon-Y release from alveolar macrophages in asthma. Am J Respir Crit Care Med 1998;157:256-62.

15. Cluzel M, Damon M, Chanez $\mathrm{P}$, et al. Enhanced alveolar cell luminol-dependent chemiluminescence in asthma. J Allergy Clin Immunol 1987;80:195-201.

16. Hew M, Bhavsar P, Torrego A, et al. Relative corticosteroid insensitivity of peripheral blood mononuclear cells in severe asthma. Am J Respir Crit Care Med 2006;174:134-41.

17. Kumar S, Boehm J, Lee JC. p38 MAP kinases: key signalling molecules as therapeutic targets for inflammatory diseases. Nat Rev Drug Discov 2003;2:717-26.

18. Koch A, Giembycz M, Ito K, et al. Mitogen-activated protein kinase modulation of nuclear factor-kappaB-induced granulocyte macrophage-colony-stimulating factor release from human alveolar macrophages. Am J Respir Cell Mol Biol 2004;30:342-9.

19. Koch A, Giembycz M, Stirling RG, et al. Effect of smoking on MAP kinase-induced modulation of IL-8 in human alveolar macrophages. Eur Respir J 2004;23:805-12.

20. Irusen E, Matthews JG, Takahashi A, et al. p38 Mitogen-activated protein kinaseinduced glucocorticoid receptor phosphorylation reduces its activity: role in steroidinsensitive asthma. J Allergy Clin Immunol 2002;109:649-57.

21. Lasa M, Abraham SM, Boucheron C, et al. Dexamethasone causes sustained expression of mitogen-activated protein kinase (MAPK) phosphatase 1 and phosphatase-mediated inhibition of MAPK p38. Mol Cell Biol 2002;22:7802-11.

22. Kassel 0, Sancono A, Kratzschmar J, et al. Glucocorticoids inhibit MAP kinase via increased expression and decreased degradation of MKP-1. EMBO J 2001;20:7108-16.

23. Chen $\mathbf{P}$, Li J, Barnes J, et al. Restraint of proinflammatory cytokine biosynthesis by mitogen-activated protein kinase phosphatase-1 in lipopolysaccharide-stimulated macrophages. J Immunol 2002;169:6408-16.
24. Zhao 0, Shepherd EG, Manson ME, et al. The role of mitogen-activated protein kinase phosphatase-1 in the response of alveolar macrophages to lipopolysaccharide: attenuation of proinflammatory cytokine biosynthesis via feedback control of p38. J Biol Chem 2005;280:8101-8.

25. Abraham SM, Clark AR. Dual-specificity phosphatase 1: a critical regulator of innate immune responses. Biochem Soc Trans 2006;34:1018-23.

26. Clark AR. MAP kinase phosphatase 1: a novel mediator of biological effects of glucocorticoids? J Endocrinol 2003;178:5-12.

27. Wenzel SE, Schwartz LB, Langmack EL, et al. Evidence that severe asthma can be divided pathologically into two inflammatory subtypes with distinct physiologic and clinical characteristics. Am J Respir Crit Care Med 1999;160:1001-8.

28. Jatakanon A, Uasuf C, Maziak W, et al. Neutrophilic inflammation in severe persistent asthma. Am J Respir Crit Care Med 1999;160:1532-9.

29. Nguyen LT, Lim S, Oates T, et al. Oral but not inhaled corticosteroid therapy increases airway neutrophils in asthma. Respir Med 2005;99:200-7.

30. Szatmary Z, Garabedian MJ, Vilcek J. Inhibition of glucocorticoid receptor-mediated transcriptional activation by p38 mitogen-activated protein (MAP) kinase. J Biol Chem 2004;279:43708-15.

31. Saccani S, Pantano S, Natoli G. p38-Dependent marking of inflammatory genes for increased NF-kappa B recruitment. Nat Immunol 2002;3:69-75.

32. Cosio BG, Mann B, Ito K, et al. Histone acetylase and deacetylase activity in alveolar macrophages and blood mononocytes in asthma. Am J Respir Crit Care Med 2004;170:141-7.

33. Dean JL, Sully G, Clark AR, et al. The involvement of AU-rich element-binding proteins in $\mathrm{p} 38$ mitogen-activated protein kinase pathway-mediated mRNA stabilisation. Cell Signal 2004;16:1113-21.

34. Sirenko 0I, Lofquist AK, DeMaria CT, et al. Adhesion-dependent regulation of an A+U-rich element-binding activity associated with AUF1. Mol Cell Biol 1997; 17:3898-906.

35. Wang SW, Pawlowski J, Wathen ST, et al. Cytokine mRNA decay is accelerated by an inhibitor of p38-mitogen-activated protein kinase. Inflamm Res 1999:48:533-8.

36. Tebo J, Der S, Frevel M, et al. Heterogeneity in control of mRNA stability by AU-rich elements. J Biol Chem 2003;278:12085-93. 